\title{
Development and in vitro evaluation of polyurethane microparticles as carrier for bevacizumab: an alternative treatment for retinopathy of prematurity
}

\author{
Ramona C Albulescu ${ }^{1}$, Florin Borcan ${ }^{2}$, Corina Paul ${ }^{1}$, Iulian Velea ${ }^{1}$, Maria Puiu ${ }^{3}$ \\ ${ }^{1} 2^{\text {nd }}$ Pediatrics Dept., Faculty of Medicine, Victor Babes University of Medicine and Pharmacy, Timisoara, Romania \\ 2 Pharm. Chem istry Dept., Faculty of Pharmacy, Victor Babes University of Medicine and Pharmacy, Timisoara, Romania \\ ${ }^{3}$ Genetics Dept., Faculty of Medicine, Victor Babes University of Medicine and Pharmacy, Timisoara, Romania
}

\begin{abstract}
Retinopathy of prematurity (ROP) is known as an abnormal development of the blood capillaries from the eyes' retina, and it is met to those infants that were born too early (premature) as its name suggests. Bevacizumab or Avastin belongs to the class of monoclonal antibodies and it is a possible treatment for those patients who have been diagnosed with a recurrent malignant glioma or other cancers due to its behavior to bind selectively to vascular endothelial growth factor (VEGF). In this study, eye drops based on a polyurethane carrier used for bevacizumab were obtained and the degradation of this carrier was studied for three weeks in two different media: simulated body fluid (SBF) and phosphate buffered saline (PBS); the influence of ultrasounds on the degradat ion of polyurethane carrier was also evaluated. The obtained results suggest that the polyurethane carrier presents a very slow degra dation which is beneficial for drugs which require low release rates and on the other hand, the concentrations of the degradation products remain at a low level.
\end{abstract}

Key Words: Bevacizumab, Polyurethane Carrier, Simulated Body Fluid, Phosphate Buffered Saline, Ultrasound.

\section{INTRODUCTION}

Retinopathy of prematurity (ROP) is a disease that occurs by altering vasoproliferative retinal vasculogenesis and this development contributes a multitude of factors (Wilson et al., 2013). The disease may present mild or may progress to retinal detachment and ultimately lead to blindness. For a long time this disorder has been considered one of the most terrible complications of prematurity, but modern treatment and prevention techniques used lately made great progress leading to a decrease in the number of cases of blindness in these children (Begue and Lopez, 2003).

ROP prevalence ranges from $5-8 \%$ in developed countries with adequate neonatal facilities and up to $30 \%$ in developing countries with average incomes. There is growing evidence that blindness due to ROP and ROP itself represent important health problems of people from middle-income countries from Latin America, Eastern Europe and in the advanced economies of Middle East and Southeast Asia. In this part of world, ROP is considered the most common cause of blindness in childhood. ROP is likely to become a growing problem in India, China and other Asian countries as these countries expand their providing care for premature babies (Di Fiore et al., 2010; Zin and Gole, 2013; Liu et al., 2012).

Retinopathy of prematurity is a debilitating disease that involves high costs for society if it is neglected. Therefore prevention, detection and early treatment confers especially premature babies and their families a decent life (Kretzer and Hittner, 1988; Gilbert, 2008).

Undeveloped retina of a premature is susceptible to insults that disrupt the neurovascular growth and thus leading to ROP (Hellström et al., 2013; Fulton et al., 2009).
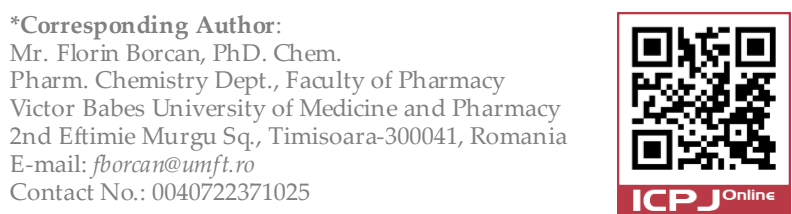

It was reported that ROP and cortical blindness are the most common causes of blindness in premature infants (Garcia-Serranoa et al., 2013). L. Filippi and his research team studied the safety and the efficiency of propranolol orally administrated to premature babies in early phase of ROP. They found that this cure is effective in counteracting the progression of ROP, but on the other hand numerous adverse effects were reported and this is the reason why the systemic administration was not considered sufficiently safe (Filippi et al., 2013). The effect of fishoil and soybean oil lipid emulsions on the development of ROP was compared in a recent paper; it was discovered that fish-oil lipid emulsions may be preventive for the development of ROP, but multicenter studies are needed to assess this effect in premature infants (Beken et al., 2014).

One year ago, a very large group of pediatric ophthalmologists from Spain published a protocol for the treatment of ROP; they presented the diode laser photocoagulation as the treatment of choice, and the fact that cryotherapy can be an alternative in the following circumstances: (i) poor mydriasis or opacities when laser therapy is impossible, (ii) as helpful technique to the treatment with laser in the case of persistent neovascularizations which can benefit from cryotherapy application, and (iii) urgent cases of aggressive ROP which require urgent treatment, but a laser is not available. Bevacizumab is known as the most widely used drug in prematures, even if several other anti-VEGF drugs have been approved such as pegaptanib, ranibizumab, etc. (Ferrer Novella et al., 2013).

The eye drops, an alternative to normal tears, are used when the body does not produce a sufficient amount of tears or the tear does not have the proper ratio of components; in the both cases resulting dry eyes (ReedKane et al., 2009). JG Crowston presents the following advantages of treatments with eye drops: non-invasive, reversible, generally safe, effective at reducing eye pressure, different mechanisms of action, and possibility 
to be additive (The Centre for Eye Research Australia, http://www.cera.org.au). Eye drops are also used for the treatment of several eye diseases (Stephen Foster, 2003). A novel approach of treating eye disease is represented by the autologous serum eye drops (ASE), but ASE producing procedures which were published are incomplete or vary significantly (Geerling et al., 2004). ASE are based on the clear part of patient's blood (serum), which is diluted with saline and bottled; it need to be kept in a freezer because it not contain any additives or preservatives (Autologous Serum Eyedrops, http://hospital.blood.co.uk/library/pdf). It should, however, be noted that practical difficulties and theoretical risks in producing ASE were reported; it was discovered that ASE are not prepared according to the international principles of collecting and preparing of blood products (Noble et al., 2004).

The applications of polyurethane materials have grown exponentially in the last century. Initially, the polyurethanes were developed in Europe as flexible fibers and foams (Shelke et al., 2014), and in the last 40 years, they have also been used in biomedical appliances or devices due to their biocompatibility and their mechanical flexibility (Cherng et al., 2013). The enormous increase of researchers' interest for polyurethane materials is due to the following reasons: (i) the rigidity of the material can be easily adjusted by modifying the order of magnitude of the molecular weight of polyols used in the synthesis (Król, 2007); (ii) the size of macromolecular structures can be easily adjusted by modifying the quantity of chain extenders which are used in the synthesis (diols with low molecular weight, as mono-ethylene glycol, 1,4butanediol, 1,6-hexanediol) (Ho et al., 1999); (iii) the stability or degradation of polyurethane materials in aqueous media can be changed by adjusting the ratio of polyester / polyether used as raw materials (UrgunDemirtas et al., 2007).

The aim of this research is to evaluate the degradation of a polyurethane carrier used for bevacizumab in order to evaluate drug release rate.

\section{MATERIALS AND METHODS}

There were used monoethylene glycol (MEG) from LachNer (Czech R.) and 1,4-butanediol (1,4-BD) from Carl Roth (Germany). Isophorone diisocyanate (IPDI), polyethylene glycol (PEG 200), polycaprolactone diol (PCL 530), acetone, and Tween ${ }^{\circledR} 20$ were purchased from Merck (Germany). The substances were not subject to any prior purification. Bevacizumab $\left(25 \mathrm{mg} / \mathrm{ml}\right.$, Avastin ${ }^{\circledR}$, Roche Diagnostics, Switzerland) was obtained from local pharmacy network.

Anhydrous salts $\left(\mathrm{NaCl}, \mathrm{NaHCO}_{3}, \mathrm{Na}_{2} \mathrm{HPO}_{4}, \mathrm{KCl}\right.$, $\mathrm{KH}_{2} \mathrm{PO}_{4}, \mathrm{~K}_{2} \mathrm{HPO}_{4}, \mathrm{MgCl}_{2}$ ) and $\mathrm{HCl}$ solution $1 \mathrm{M}$ were used to obtain SBF and PBS. The inorganic salts (analytical grade, $<1.0 \%$ insoluble impurities) were heated at $110{ }^{\circ} \mathrm{C}$ for one hour previously any other operation in order to remove any traces of crystallization water.

\section{Eye drops' preparation}

Bevacizumab was encapsulated inside polyurethane microparticles (PuMp) in oder to obtain eye drops with a slow drug release rate. PuMp were synthesized in a multistep process described in Figure 1. Initially two phases were magnetically stirred $(400 \mathrm{rpm})$ and preheated at $40^{\circ} \mathrm{C}$ separately: the isocyanate phase contains $0.6 \mathrm{ml}$ IPDI was dissolved in $20 \mathrm{ml}$ acetone; the aqueous phase was a mixture of $0.6 \mathrm{~mL}$ MEG, $0.6 \mathrm{~mL}$ 1,4-BD, $1.2 \mathrm{~mL}$ PEG 200, $1.5 \mathrm{~mL}$ Tween ${ }^{\circledR 2} 2$, and $40 \mathrm{~mL}$ distilled water. In the

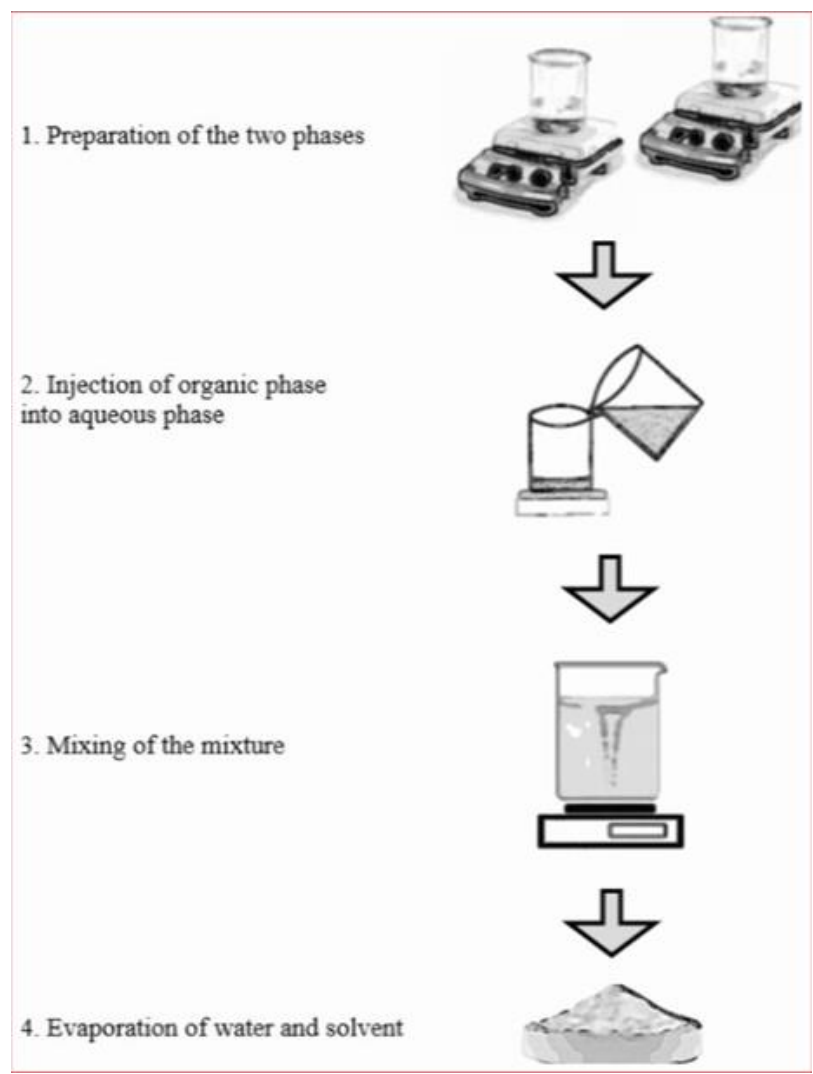

Figure 1: The steps of PuMp and PeuMp synthesis.

second step, the organic phase was rapidly injected in aqueous phase using a $1 / 1.1(\mathrm{v} / \mathrm{v})$ ratio between the two phases. An excess of aqueous phase was preferred because: (i) it can be easily removed when the products are washed; (ii) it assures a complete reaction of all isocyanate groups and thus prevent the formation of secondary products (amines from the reaction between unreacted isocyanate groups and water). The mixture was magnetically stirred $(600 \mathrm{rpm})$ for 4 hours to assure the finishing of all chemical reactions; this was the step when PuMp are formed. In the last step, the products were washed with distilled water for three times and there were dried in a laboratory oven at $110^{\circ} \mathrm{C}$ for 12 hours.

Bevacizumab was added previous to formation of PuMp walls in the first step of the synthesis. The drug was used as a solution $25 \mathrm{mg} / \mathrm{ml}$ (bevacizumab, sodium phosphate, Tween ${ }^{\circledR 20}$, and water) and added in the aqueous phase. A quantity of $0.1 \mathrm{ml}$ bevacizumab solution was injected into $2.4 \mathrm{ml}$ active components of aqueous phase.

The dried PuMp powder with bevacizumab was dissolved in a saline aqueous solution $1 \%$ in order to obtain the eye drops. It was chosen a 1:5000 w/v dilution based on our experience about the polyurethane nano- and micro-structures' solubility (Borcan et al., 2012).

The same procedure was used to prepare the positive control groups (polyester-urethane microparticles, PeuMp samples), but polyethylene glycol as raw material was replaced with polycaprolactone diol; same quantity was used. Polyester-urethane microparticles were chosen due to their increased water degradation by hydrolysis (Bikiaris, 2013). 
Table 1: The treatments' scheme used to study the degradation of polyurethane carrier.

\begin{tabular}{|c|c|c|c|}
\hline Sample code & Sample description & $\begin{array}{c}\text { Degradation } \\
\text { medium }\end{array}$ & $\begin{array}{c}\text { Degradation } \\
\text { treatment }\end{array}$ \\
\hline PuMp_1 & & SBF & stirring \\
\hline PuMp_2 & PuMp with $2.5 \mathrm{mg}$ bevacizumab, dissolved in saline aqueous & PBS & stirring \\
\hline PuMp_3 & solution $1 \%(1: 5000, \mathrm{w} / \mathrm{v})$ & SBF & ultrasound \\
\hline PuMp_4 & & PBS & ultrasound \\
\hline PuMp_01 & & SBF & stirring \\
\hline PuMp_02 & PuMp without bevacizumab, dissolved in saline aqueous & PBS & stirring \\
\hline PuMp_03 & solution $1 \%(1: 5000, \mathrm{w} / \mathrm{v})$, used as negative control & SBF & ultrasound \\
\hline PuMp_04 & & PBS & ultrasound \\
\hline PeuMp_01 & & SBF & stirring \\
\hline PeuMp_02 & PeuMp without bevacizumab, dissolved in saline aqueous & PBS & stirring \\
\hline PeuMp_03 & solution $1 \%(1: 5000, \mathrm{w} / \mathrm{v})$, used as positive control & SBF & ultrasound \\
\hline PeuMp_04 & & PBS & ultrasound \\
\hline
\end{tabular}

Table 2: Evolution of carriers' size during the experiment.

\begin{tabular}{cccc}
\hline \multirow{2}{*}{ Sample code } & \multicolumn{3}{c}{ Carriers' size (nm) \pm SE vs. Period of degradation treatment (days) } \\
\cline { 2 - 4 } & $\mathbf{5}$ & $\mathbf{1 0}$ & $\mathbf{1 5}$ \\
\hline PuMp_1 & $682 \pm 36$ & $680 \pm 44$ & $676 \pm 28$ \\
PuMp_2 & $675 \pm 44$ & $671 \pm 36$ & $673 \pm 45$ \\
PuMp_3 & $687 \pm 37$ & $684 \pm 43$ & $680 \pm 26$ \\
PuMp_4 & $668 \pm 33$ & $665 \pm 46$ & $663 \pm 35$ \\
PuMp_01 & $681 \pm 26$ & $677 \pm 43$ & $679 \pm 27$ \\
PuMp_02 & $670 \pm 35$ & $668 \pm 36$ & $671 \pm 34$ \\
PuMp_03 & $669 \pm 34$ & $668 \pm 42$ & $665 \pm 43$ \\
PuMp_04 & $673 \pm 44$ & $670 \pm 45$ & $669 \pm 43$ \\
PeuMp_01 & $643 \pm 28$ & $521 \pm 36$ & $404 \pm 39$ \\
PeuMp_02 & $659 \pm 36$ & $538 \pm 38$ & $442 \pm 27$ \\
PeuMp_03 & $651 \pm 37$ & $517 \pm 40$ & $409 \pm 36$ \\
PeuMp_04 & $648 \pm 45$ & $524 \pm 47$ & $678 \pm 29$ \\
\end{tabular}

\section{Obtaining of the degradation media}

The protocol to prepare the simulated body fluid (SBF) was described in the literature long time ago. It is a solution containing the following ions at approximately the same concentrations as the human plasma: $\mathrm{Na}^{+}, \mathrm{K}^{+}$, $\mathrm{Mg}^{2+}, \mathrm{Ca}^{2+}, \mathrm{Cl}^{-}, \mathrm{HCO}_{3}{ }^{-}, \mathrm{HPO}_{4}{ }^{2-}$, and $\mathrm{SO}_{4}{ }^{2-}$. A $500 \mathrm{ml}$ flat bottom flask was washed with a neutral detergent and respectively with $\mathrm{HCl}$ solution $1 \mathrm{M}$. Distilled water and salts previously presented were added and the mixture was magnetically stirred and heated to 36.5 degree with a VELP heating magnetic stirrer. The mixture was buffered at $\mathrm{pH} 7.4$ with tris-hydroxymethyl-aminomethane solution $0.05 \mathrm{M}$ and $\mathrm{HCl}$ solution $0.045 \mathrm{M}$ (Kokubo et al., 1990).

Phosphate buffered saline (PBS) was used as the second degradation medium. The medium was prepared by mixing $61.1 \mathrm{ml}$ solution $\mathrm{A}$ and $38.9 \mathrm{ml}$ solution $\mathrm{B}$ and finally adding $0.85 \mathrm{~g} \mathrm{NaCl}$. The salts-water mixtures were slowly stirred in order to avoid the introducing of oxygen into solutions. Solution A is a $\mathrm{Na}_{2} \mathrm{HPO}_{4}$ solution $0.067 \mathrm{M}$ (947 $\mathrm{mg}$ in $100 \mathrm{ml}$ distilled water), while solution B is a $\mathrm{KH}_{2} \mathrm{PO}_{4}$ solution $0.067 \mathrm{M}(907 \mathrm{mg}$ in $100 \mathrm{ml}$ distilled water) (Azger Dusthackeera et al., 2012). The pH of PBS was adjusted to 7.4 with $\mathrm{HCl}$. Both media were finally sterilized by autoclaving.

\section{Ultrasound treatment}

The two media containing the eye drops were divided in two parts: one of them was subjected to an ultrasound treatment and the other one was only magnetically stirred without any heating for four hours daily (table 1).

A powerful ultrasonic lab homogenizer UP200S (Hielscher, Germany) was used for the ultrasound treatment. The device is suited for all ultrasonic applications in small scale: homogenization, disintegration, emulsification or cell disruption (UP200H/UP200S, http://www.hielscher.com). There were chosen the following settings of the device: complete cycles, an average amplitude of the oscillatory system (50\%), and the S3 (micro tip 3) standard sonotrode.

A zetasizer (Malvern Instruments, UK) was used to evaluate the size of PuMp and PeuMp. For this purpose, the same aqueous solutions $(1: 5000 \mathrm{w} / \mathrm{v})$ were used; the measurements were repeated three times for each sample. The results were used to estimate the degradation rate of the samples.

\section{Compliance with ethics requirements}

Authors declare that they have no conflict of interest and there were not any procedures involving animal or human subjects in this research.

\section{Statistical analysis}

For the statistical analysis of the obtained data, it was used the Analysis ToolPak from MS Excel Add-ins. The data are presented as average values. The correlation between features was evaluated using the Spearman rank coefficient $\mathrm{r}$. P values less than 0.05 were considered statistically significant.

\section{RESULTS AND DISCUSSION}

The zetasizer results about the evolution of PuMp and PeuMP size during the experiment are shown in Table 2. It is important to mention that PuMp (sample with bevacizumab) presented an average size of $679 \pm 48 \mathrm{~nm}$ and a zeta potential of $28.1 \pm 0.6 \mathrm{mV}$ prior any degradation treatment, PuMp_0 (sample of empty particles, without bevacizumab) presented an average size of $682 \pm 39 \mathrm{~nm}$ and a zeta potential of $28.4 \pm 0.2 \mathrm{mV}$, while PeuMp presented 
Table 3: The equations and the slopes of samples' degradation trendlines

\begin{tabular}{cccc}
\hline Sample code & $\begin{array}{c}\text { Equation of } \\
\text { trend lines }\end{array}$ & $\begin{array}{c}\text { Slope of } \\
\text { trend lines }\end{array}$ & P values \\
\hline PuMp_1 & $\mathrm{y}=-0.26 \cdot \mathrm{x}+682.5$ & -0.26 & 0.014 \\
PuMp_2 & $\mathrm{y}=-0.14 \cdot \mathrm{x}+674.5$ & -0.14 & 0.007 \\
PuMp_3 & $\mathrm{y}=-0.38 \cdot \mathrm{x}+688.0$ & -0.38 & 0.001 \\
PuMp_4 & $\mathrm{y}=-0.28 \cdot \mathrm{x}+668.5$ & -0.28 & 0.007 \\
PuMp_01 & $\mathrm{y}=-0.14 \cdot \mathrm{x}+680.5$ & -0.14 & 0.003 \\
PuMp_02 & $\mathrm{y}=-0.12 \cdot \mathrm{x}+670.5$ & -0.12 & 0.007 \\
PuMp_03 & $\mathrm{y}=-0.18 \cdot \mathrm{x}+669.5$ & -0.18 & 0.001 \\
PuMp_04 & $\mathrm{y}=-0.20 \cdot \mathrm{x}+673.0$ & -0.20 & 0.003 \\
PeuMp_01 & $\mathrm{y}=-18.24 \cdot \mathrm{x}+714.5$ & -18.24 & 0.014 \\
PeuMp_02 & $\mathrm{y}=-19.32 \cdot \mathrm{x}+743.5$ & -19.32 & 0.028 \\
PeuMp_03 & $\mathrm{y}=-19.86 \cdot \mathrm{x}+731.5$ & -19.86 & 0.028 \\
PeuMp_04 & $\mathrm{y}=-19.40 \cdot \mathrm{x}+729.0$ & -19.40 & 0.007 \\
\hline
\end{tabular}

an initial average size of $825 \pm 31 \mathrm{~nm}$ and a zeta potential of 27.2 $\pm 0.4 \mathrm{mV}$. An important difference between the size of PuMp and PeuMp samples was recorded; this is due to the difference of polyols' molecular weight - PEG with M 200 and PCL with M 530 were used as raw materials.

It is simple to observe that the trendline of all four degradation treatments is an easier decrease of PuMp size. However, it is important to make differences between the slopes of the trendlines. The trendlines' equations and slopes were extracted from the MS Excel for this purpose and there are presented in Table 3.

How much a straight line deviates from the horizontal axis is one of its most important characteristics; this parameter is called the "slope" of the straight line (Slope of a Straight Line, http://www.purplemath.com/modules/slope.htm). In our experiment there were obtained similar slopes of degradation trend lines (around -0.2), except the PuMp_3 sample. In conclusion, eye drops containing polyurethane microparticles with bevacizumab present a very slight degradation in the first 20 days, but this process can be accelerated with an ultrasound treatment (the samples treated with ultrasounds showed higher slopes in both media).

Important degradations were observed for all four treatments in the case of PeuMp sample used as positive control; the slopes of trend lines present values between 18.24 and -19.86 . The same accelerated degradations was obtained for PeuMp sample treated with ultrasounds.

The empty PuMp presented very slight degradation in the first 20 days, with slopes of trend lines between 0.12 and -0.20 ; these values are closed to those obtained for PuMp with bevacizumab indicating that the drug not influence the microparticles' degradation.

The carrier degradation rate on the one side, and the bevacizumab release rate on the other side, is very important because: (i) bevacizumab presents a short halflife (Li et al., 2012), (ii) a slow degradation of a carrier assures a prolonged drug delivery, and (iii) it assure low concentrations of degradation products in patient body.

Another important aspect of the obtained PuMp is how the polymer particles fixed the drug. The synthesis procedure (a complex process consisting of a polyaddition reaction and a simultaneous emulsification) tends to ensure the formation of hollow polymer particles which physically encapsulate the drug, but in our case the bevacizumab macrostructure presents reactive functional groups. This is the reason why it is assumed that between the drug and the carrier appear chemical interactions too.
This chemical bonds decrease the bevacizumab release rate.

It is important to mention that other polymer carriers reported in literature also present very slow degradations. The degradation of bevacizumab-loaded PLGA nanoparticles was studied (Li et al., 2012) during a 100 days experiment. It was found that polymer carriers' degradation rate increases when the molar mass decreases (Lao et al., 2011); the carriers' size can be easily adjusted to lower values and thus can easily penetrate through cornea but the yield of encapsulation decreases when the carriers' size decreases. In polyurethane domain, the degradation of structural chains in aqueous media can be easily adjusted by inserting of polyester in detrimental of the polyether used as raw material (Rychlý et al., 2011).

\section{CONCLUSION}

Polyurethane microparticles used as carrier for bevacizumab were obtained through a microemulsion process. The microparticles were synthesized using a minimum addition of chemical substances: it was not used any catalyst although the polyurethane chemistry uses tertiary amines and/or organotin compounds and on the other hand, the quantity of surfactant has been minimized, however, the microparticles' stability was not be influenced. Eye drops based on polyurethane microparticles were obtained and there were placed in two different media (simulated body fluid and phoasphate buffered saline) and there were also subjected to an ultrasound treatment. The degradation was evaluated by comparing of the average size of polyurethane microparticles. It was found that degradation of the polymer carriers is very slow, which it is benefit to a slow bevacizumab release and to minimize the concentration of degradation products in patient body.

\section{ACKNOWLEDGEMENT}

F. Borcan was financially supported by Victor Babes University of Medicine and Pharmacy, Timisoara, Romania (internal competition grant no. 15250/2012).

\section{REFERENCES}

Azger Dusthackeera, V.N., Nagarajana, P., Dasb, D., Kumara, V. and Selvakumar, N. (2012). Retrieval of Mycobacterium tuberculosis cultures suspended in phosphate buffered saline. Int J Mycobacteriol, Volume 1, Pages 149-151. [DOI]

Begue, N.M., Lopez, J.P. (2003). Retinopatía del prematur o: incidencia, gravedad y evolución. Anal Pediatr, Volume 58, Pages 156-161. [DOI]

Beken, S., Dilli, D., Fettah, N.D., Kabataș, E.U., Zenciroğlu, A. and Okumuş, N. (2014). The influence of fish-oil lipid emulsions on retinopathy of prematurity in very low birth weight infants: A randomized controlled trial. Early Hum Dev, Volume 90, Pages 27-31. [DOI]

Bikiaris, D.N. (2013). Nanocomposites of aliphatic polyesters: An overview of the effect of different nanofillers on enzymatic hydrolysis and biodegradation of polyesters. Polym Degr Stab, Volume 98, Pages 19081928. [DOI]

Borcan, F., Soica, C.M., Ganta, S., Amiji, M.M., Dehelean, C.A. and Munteanu, M.F. (2012). Synthesis and preliminary in vivo evaluations of polyurethane microstructures for transdermal drug delivery. Chem Cent J, Volume 6, Pages 1-10. [DOI]

Cherng, J.Y., Hou, T.Y., Shih, M.F., Talsma, H. and Hennink, W.E. (2013) Polyurethane-based drug delivery systems. Int J Pharm, Volume 450, Pages 145-162. [DOI]

Di Fiore, J.M., Bloom, J.N., Orge, F., Schutt, A., Schluchter, M., Cheruvu, V.K., Walsh, M., Finer, N., Martin, R.J. (2010). A Higher Incidence of Intermittent Hypoxemic Episodes Is Associated with Severe Retinopathy of Prematurity. J Pediatr, Volume 157, Pages 69-73. [DOI]

Ferrer Novella, C., González Viejo, I., Pueyo Royo, V., Martínez Fernández R., Galdós Iztueta, et al. (2013). A protocol for the treatment of retinopathy of prematurity in Spain. Ardh Soc Esp Oftalmol, Volume 88, Pages 231-236. [DOI] 
Filippi, L., Cavallaro, G., Bagnoli, P., Dal Monte, M., Fiorini, P., et al. (2013) Oral Propranolol for Retinopathy of Prematurity: Risks, Safety Concerns, and Perspectives. J Pediatr, Volume 163, Pages 1570-1577. [DOI]

Fulton, A.B., Hansen, R.M., Moskowitz, A., Akula, J.D. (2009). The neurovascular retina in retinopathy of prematurity. Prog Retin Eye Res, Volume 28, Pages 452-482. [DOI]

Garcia-Serranoa, J.L., Uberos Fernándezb, J., Anaya-Alaminosa, R., JerezCalerob, A., Padilla-Torresa, J.F., Ramírez-Garciac, M.C., Pi-ar-Molina, R. (2013). "Oxygen with Love" and Diode Laser Treatment Decreases Comorbidity and Avoidable Blindness due to Retinopathy of Prematurity: Results Achieved in the Past 12 Years. Ped Neonat, Volume 54, Pages 397-401. [DOI]

Geerling, G., MacLennan, S. and Hartwig, D. (2004). Autologous serum eye drops for ocular surface disorders. Br J Ophthalmol, Volume 88, Pages 1467-1474. [DOI]

Gilbert, C. (2008). Retinopathy of prematurity: a global perspective of the epidemics, population of babies at risk and implications for control. Early Hum Dev, Volume 84, Pages 77-82. [DOI]

Hellström, A., Smith, L.E.H. and Dammann, O. (2013). Retinopathy of prematurity. Lancet, Volume 382, Pages 1445-1457. [DOI]

Ho, K.S., Hsieh, K.H., Huang, S.K. and Hsieh, T.H. (1999). Polyurethanebased conducting polymer blends: I. Effect of chain extender. Synt Met, Volume 107, Pages 65-73. [DOI]

Kokubo, T., Kushitani, H., Sakka, S., Kitsugi, T. and Yamamuro, T. (1990). Solutions able to reproduce in vivo surface-structure changes in bioactive glass-ceramic A-W. J Biomed Mater Res, Volume 24, Pages 721-734. [DOI]

Kretzer, F.L. and Hittner, H.M. (1988). Retinopathy of prematurity: clinical implications of retinal development. Arch Dis Child, Volume 63, Pages 1151-1167. [DOI]

Król, P. (2007). Synthesis methods, chemical structures and phase structures of linear polyurethanes. Properties and applications of linear polyurethanes in polyurethane elastomers, copolymers and ionomers. Prog Mat Sci, Volume 52, Pages 915-1015. [DOI]

Lao, L.L., Peppas, N.A., Boey, F.Y. and Venkatraman, S.S. (2011). Modeling of drug release from bulk-degrading polymers. Int J Pharm, Volume 418, Pages 28-41. [DOI]
Li, F., Hurley, B, Liu, Y, Leonard, B and Griffith, M. (2012). Controlled Release of Bevacizumab Through Nanospheres for Extended Treatmen of Age-Related Macular Degeneration. Open Ophthalmol J, Volume 6, Pages 54-55. [DOI]

Liu, Y.-S., Chen, T.-C., Yang, C.-H., Yang, C.-M., Huang, J.-S., Ho, T.-C., Chen, M.-S. (2012). Incidence, risk factors, and treatment of retinopathy of prematurity among very low birth body weight infants. Taiwan J Ophthal, Volume 2, Pages 60-63. [DOI]

Noble, B.A., Loh, R.S.K., MacLennan, S., Pesudovs, K., Reynolds, A., Bridges, L.R., Burr, J., Stewart, O. and Quereshi, S. (2004) Comparison of autologous serum eye drops with conventional therapy in a randomised controlled crossover trial for ocular surface disease. Br J Ophthalmol, Volume 88, Pages 647-652. [DOI]

Reed-Kane, D., Carlson, R.A., Kupiec, T.C. and Vu, N. (2009). Applications and Sterility of Autologous Serum Eye Drops. Int J Pharm Compound, Volume 13, Pages 540-543.

Rychlý, J., Lattuati-Derieux, A., Lavédrine, B., Matisová-Rychlá, L. Malíková, M., Csomorová, K. and Janigová, I. (2011). Assessing the progress of degradation in polyurethanes by chemiluminescence and thermal analysis. II. Flexible polyether- and polyester-type polyurethane foams. Polym Degr Stab, Volume 96, Pages 462-469. [DOI]

Shelke, N.B., Nagarale, R.K., Kumbar, S.G. (2014). Chapter 7 - Polyurethanes. In S.G. Kumbar, C. Laurencin, M. Meng Deng. Natural and Synthetic Biomedical Polymers (pp. 123-144). Burlington, MA: Elsevier Inc. [DOI]

Stephen Foster, C. (2003). Systemic Treatment of Ocular Disease. In J. Zeiss. Essential Guide to Behcet's disease (pp. 105-106). Uxbridge, MA: Central Vision Press.

Urgun-Demirtas, M., Singh, D. and Pagilla, K. (2007). Laboratory investigation of biodegradability of a polyurethane foam unde anaerobic conditions. Polym Degr Stab, Volume 92, Pages 1599-1610. [DOI]

Wilson, C.M., Ells, A.L., Fielder, A.R. (2013). The Challenge of Screening for Retinopathy of Prematurity. Clin Perinatol, Volume 40, Pages 241259. [DOI]

Zin, A., Gole, G.A. (2013). Retinopathy of Prematurity-Incidence Today. Clin Perinatol, Volume 40, Pages 185-200. [DOI] 